\title{
Incidence and Predictors of Dysphagia Following Anterior Cervical Discectomy and Fusion: Prospective Observational Study
}

\author{
Yingda Li1 ${ }^{1}$, Kerry Hitos ${ }^{2}$, Su-Thet Mon', Natalie Knight ${ }^{3}$, Nick Vrodos ${ }^{1}$ \\ ${ }^{1}$ Departments of Neurosurgery, Flinders Medical Centre, Adelaide, Australia \\ ${ }^{2}$ Department of Surgery, The University of Sydney, Westmead Hospital, Sydney, Australia \\ ${ }^{3}$ Departments of Speech Pathology, Flinders Medical Centre, Adelaide, Australia \\ Email: dryingdali@gmail.com
}

How to cite this paper: Li, Y., Hitos, $\mathrm{K}$. Mon, S.-T., Knight, N. and Vrodos, N. (2018) Incidence and Predictors of Dysphagia Following Anterior Cervical Discectomy and Fusion: Prospective Observational Study. Open Journal of Modern Neurosurgery, 8, 132-141.

https://doi.org/10.4236/ojmn.2018.81011

Received: December 30, 2017

Accepted: January 27, 2018

Published: January 30, 2018

Copyright $(9) 2018$ by authors and Scientific Research Publishing Inc. This work is licensed under the Creative Commons Attribution International License (CC BY 4.0).

http://creativecommons.org/licenses/by/4.0/

\begin{abstract}
The incidence of dysphagia following anterior cervical discectomy and fusion (ACDF) reported in the literature varies due to differences in measures and time intervals applied, ranging from nearly $3 / 4$ at 2 weeks to $13 \%$ at 12 months. The most commonly used dysphagia scales remain subjective, nonvalidated, and do not capture functional impact. Various risk factors have been identified, though few consistently reproduced and none studied in an Australasian context. The aims of this study were to use objective and validated measures, assess both traditional and novel risk factors, and determine the impact of dysphagia on dietary status, length of stay and complications. METHODS: Twenty-nine adults undergoing ACDF for degenerative pathologies were enrolled between March and November 2015 in an Australian institution. Bazaz dysphagia scale, Dysphagia short questionnaire, 3-ounce swallow test and dietary status were assessed preoperatively, and 2 days and 6 weeks postoperatively. Descriptive statistics were used to characterise the study sample and logistic regression modelling performed on risk factors. RESULTS: Dysphagia incidence ranged from $85 \%$ on day-2 Dysphagia short questionnaire to zero on 6-week 3-ounce swallow. All measures increased at 2 days and fell by 6 weeks. Failing day-2 3-ounce swallow was the only measure associated with modifications in solids $(P=0.06)$, showing significant linear correlation $(\mathrm{P}=0.02)$. Respiratory comorbidity increased risk of failing day-2 3-ounce swallow by more than 32-fold (OR: 32.4; 95\% CI: 1.8 - 587.7; P = 0.019) and scoring moderate or severe on Bazaz by almost 10-times (OR: 9.3; 95\% CI: 0.9 - 95.95; P = 0.061). Psychiatric history also increased risk of failing day-2 3-ounce swallow by more than 10-fold (OR: 10.9; 95\% CI: 1 - 123.7; $\mathrm{P}=0.054$ ). Failing 3-ounce swallow increased length of stay (5 versus 7 days;
\end{abstract}


$\mathrm{P}=0.013)$. CONCLUSIONS: Incidence of dysphagia following ACDF was dependent on the measure used, though all improved over time. The 3-ounce swallow test correlated with dietary modification and length of stay, while respiratory comorbidity posed the most significant risk. This may identify a group of patients who may benefit from early intervention.

\section{Keywords}

Dysphagia, ACDF, Incidence, Predictors

\section{Introduction}

\subsection{Incidence}

Anterior cervical discectomy and fusion (ACDF) is one of the most commonly performed operations on the spine. While complication rates are relatively low, ACDF can be associated with specific risks, including dysphagia, leading to aspiration risk, dietary modification, and even nasogastric feeds or gastrostomy in the severest of cases. Furthermore, postoperative dysphagia has been associated with increased length of stay (LOS), readmission rate, in-hospital costs and mortality [1]. Dysphagia incidence reported in the literature varies widely due to differences in measurement tools used and time intervals applied, with rates of up to $71 \%$ at 2 weeks [2], gradually decreasing but persistent in up to $13 \%$ at 12 months [3]. While potentially posing the highest aspiration risk and thus providing an opportunity for early intervention, the incidence and severity of immediate postoperative dysphagia (within the first few days) remain unclear.

\subsection{Measurement Tools}

The most commonly used scale in the literature, the Bazaz dysphagia score [3], is subjective, remains non-validated and does not capture functional impact. On the other hand, validated questionnaires such as the quality of life in swallowing disorders survey (SWAL-QOL) [4] and the MD Anderson dysphagia inventory [5] are complex and serve mainly as research tools. While fibreoptic endoscopy and videofluoroscopy are considered the gold standards, they are invasive, expensive and labour-intensive. The Dysphagia short questionnaire is a short 5-category survey that has recently been validated in a group of patients undergoing anterior cervical surgery [6], while the 3-ounce swallow test is a simple, objective bedside tool that has been validated against fibreoptic endoscopy in a cohort of 3000 patients with varying pathologies, with a sensitivity of over $96 \%$ for predicting aspiration status [7]. Neither of these tools has been used specifically in the postoperative ACDF setting.

\subsection{Risk Factors}

Multiple demographic, comorbid and surgical risk factors have been identified 
in the literature, though most derived from retrospective studies, few consistently reproduced, and none studied in an Australasian context. Demographic risk factors include increasing age, female gender, high body-mass index (BMI), and smoking [8]. Comorbid factors include psychiatric history, endocrinopathy [9] and preoperative neck pain, however, the role of more common comorbidities such as cardiovascular and respiratory disease remain unclear. Surgical factors include high-level, multilevel and revision surgery, operative duration, implant profile (plate vs. zero-profile), fusion (vs. arthroplasty) [10], overcorrection of kyphosis [11], and use of bone morphogenetic protein. However, the relative indications for zero-profile systems and arthroplasty remain unresolved.

Preoperative tracheal traction exercises have been identified as potentially protective, while there is conflicting evidence regarding reducing endotracheal cuff pressure following retractor placement [12] [13]. There are reports of successful dysphagia treatment by removing anterior cervical osteophytes, but not in the setting of ACDF [14]. Evidence for an association with postoperative soft tissue swelling is similarly conflicting [15], though analyses have only been performed with plain radiographs. There is randomised trial evidence that systemic and local steroids reduce postoperative dysphagia [16] [17], although concerns regarding non-union remain.

The objectives of our study were to assess the incidence, severity and natural history of postoperative dysphagia in a prospective Australasian patient cohort using validated measurement tools, determine its impact on dietary modification, length of stay and complication rate, and identify risk factors and potential targets for intervention.

\section{Methods}

\subsection{Patient Selection}

This was a prospective study. All patients undergoing ACDF for degenerative cervical spine disease between March and November 2015 were enrolled. Patients undergoing corpectomy, revision surgery or surgery for traumatic, infectious or malignant pathologies were excluded, as were patients who reported significant preoperative dysphagia. Baseline characteristics including age, gender, smoking status, BMI and specific comorbidities were recorded. Operative details including mode of presentation (emergency versus elective, myelopathy versus radiculopathy), surgical level(s), implant used and duration of surgery were assessed. Postoperatively, hospital LOS and specific complications were also recorded.

\subsection{Surgical Details}

All patients at our institution underwent a right-sided approach, with the use of self-retaining retractors and distraction pins, and cage-plate system (Atlantis plate, thickness $2.5 \mathrm{~mm}$, Medtronic Sofamor Danek, Memphis, TN, USA, and Blue Ridge plate, thickness 2.4 - 2.65 mm, K2M Group Holdings, Leesberg, VA, USA) filled with a combination of local bone and tricalcium phosphate. Bone 
morphogenetic protein was not used. Endotracheal cuffs were not routinely deflated and reinflated following retractor placement. Drains were routinely placed in the prevertebral space and removed the day after surgery. All patients underwent postoperative CT on day-1.

\subsection{Dysphagia Measurement}

The Bazaz dysphagia score, Dysphagia short questionnaire, and standardised Australian food texture and fluid viscosity scales, and the 3-ounce swallow test were administered at pre-admissions clinic, on inpatient postoperative day-2, and at 6-week follow-up. The Bazaz dysphagia score [3] categorises severity into none, mild, moderate and severe based on relative frequencies of difficulty swallowing solids and liquids. The Dysphagia short questionnaire [6] scores a patient out of 18 based on their a) ability to swallow, b) incorrect swallow, c) lump feeling, d) involuntary weight loss, and e) pneumonia. The 3-ounce swallow test [7] has the patient drink 3-ounces of water from a cup in sequential swallows without stopping. Failure is defined as inability to complete the task, or if coughing, choking or wet hoarse voice occurs during or within one minute of task completion. Standardised Australian food texture (normal, soft, minced and puree) and fluid viscosity scales (normal, mildly thick, moderately thick and extremely thick) [18] were used to assess dietary modification. Plate apposition against the vertebral body was assessed on the postoperative sagittal CT and the maximum distance between the posterior margin of the plate and the ventral vertebral surface measured in $\mathrm{mm}$.

The study protocol was approved by the institutional ethics committee. All subjects gave informed consent to participation.

\subsection{Statistical Analysis}

Descriptive statistics were used to characterise the study sample for demographic details, comorbidities, dysphagia incidence and severity, as well as operative and postoperative details. For all data normality of distribution was evaluated using the Kolmogorov-Smirnov test with Lilliefors significance correction. Continuous data is presented as median and interquartile range (IQR, range from the $25^{\text {th }}$ to the $75^{\text {th }}$ percentile). Comparisons were made using the Mann Whitney U test for continuous data and the chi-square or Fisher's exact tests for proportions. A multiple regression model with backward stepwise (likelihood ratio) variable selection was used for dysphagia risk. Where possible odds ratios (OR) and 95\% confidence intervals (CI) are presented. All analyses were performed using IBM SPSS version 22.0 (SPSS Inc., Chicago, IL, USA). All tests were two tailed and statistically significant differences were considered at the $\mathrm{P}<0.05$ level.

\section{Results}

\subsection{Patient Sample}

Twenty-nine consecutive patients planned for ACDF were enrolled. Three pa- 
tients were excluded; two underwent non-ACDF procedures following review of their clinical status and imaging (one cervical laminectomy and lateral mass fusion, the other cervical arthroplasty), the third failed to complete the questionnaires, leaving 26 patients available for day- 2 analysis. A further 5 patients were excluded from 6-week evaluation; 4 returned interstate or rurally and were lost to follow-up, the fifth was followed up privately, leaving 21 patients available for 6-week analysis.

\subsection{Demographics, Comorbidities and Mode of Presentation}

Median age was 54 years (IQR: 43 - 63). 62\% of patients were female. Median BMI was $30 \mathrm{~kg} / \mathrm{m}^{2}$ (IQR: 27.8 - 35.3) and 35\% of patients were active smokers. Cardiovascular comorbidities were present in $38 \%$ of patients, most commonly hypertension and ischaemic heart disease. Psychiatric history was present in $38 \%$, with depression and anxiety most common. In total, $23 \%$ suffered respiratory disease, most commonly asthma and obstructive sleep apnoea, and 19\% of patients had diabetes. Overall, $88 \%$ of patients were treated electively and $12 \%$ as emergencies. Half of the patients presented with radiculopathy while the other $50 \%$ suffered myelopathy.

\subsection{Operative Details and Plate Apposition}

In total, $54 \%$ were multilevel surgeries. $\mathrm{C} 5 / 6+6 / 7 \mathrm{ACDF}$ was the most common level operated on (35\%), followed by C3/4 (15\%), C4/5 + 5/6 (15\%), and C6/7 (15\%). The median operative duration was $195 \mathrm{~min}$ (IQR: 158.8 - 243.3). The median distance between plate and vertebral body was $1.5 \mathrm{~mm}$ (IQR: 1 - 2.6).

\subsection{Length of Stay and Complications}

The median hospital LOS was 5 days (IQR: 3 - 7). Approximately $4 \%$ of patients had wound complications ( 1 seroma), $8 \%$ respiratory complications ( 1 atelectasis, 1 pneumonia), and $12 \%$ urinary tract infections.

\subsection{Dysphagia Incidence and Severity (Figure 1 and Figure 2)}

On day-2, dysphagia incidence ranged from as low as $31 \%$ on modified solids or liquids to $85 \%$ on Dysphagia short questionnaire. At 6 weeks, dysphagia ranged from zero on 3-ounce swallow test to $67 \%$ on Dysphagia short questionnaire. Similarly, day- 2 dysphagia severity ranged from $8 \%$ on pureed diet to $31 \%$ scoring severe on Bazaz, falling at 6 weeks to zero on pureed diet while $38 \%$ remained moderately dysphagic on Bazaz. Trends in Bazaz dysphagia scores were closely mirrored by Dysphagia short questionnaire, while dietary status was mirrored by 3 -ounce swallow.

\subsection{Correlation between Different Dysphagia Measures and Dietary Modification}

Failing day-2 3-ounce swallow test was the only measure associated with solid 


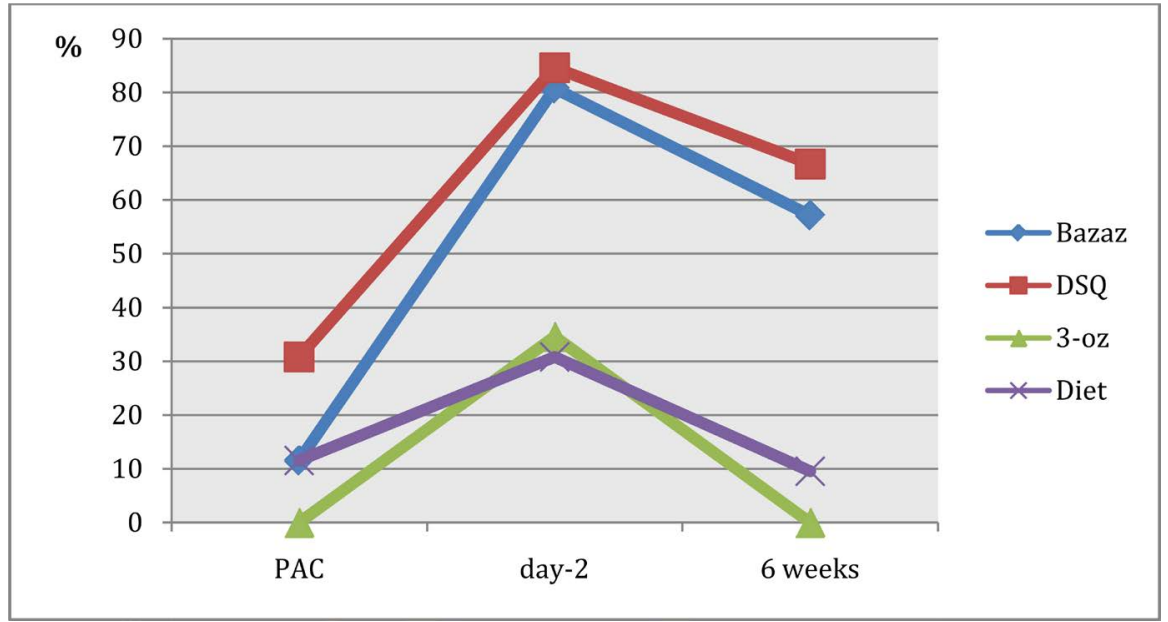

DSQ: Dysphagia short questionnaire. 3-oz: 3-ounce swallow test. PAC: Pre-admissions clinic.

Figure 1. Incidence of dysphagia based on the 4 different measurement tools, expressed as percentage of total patients.

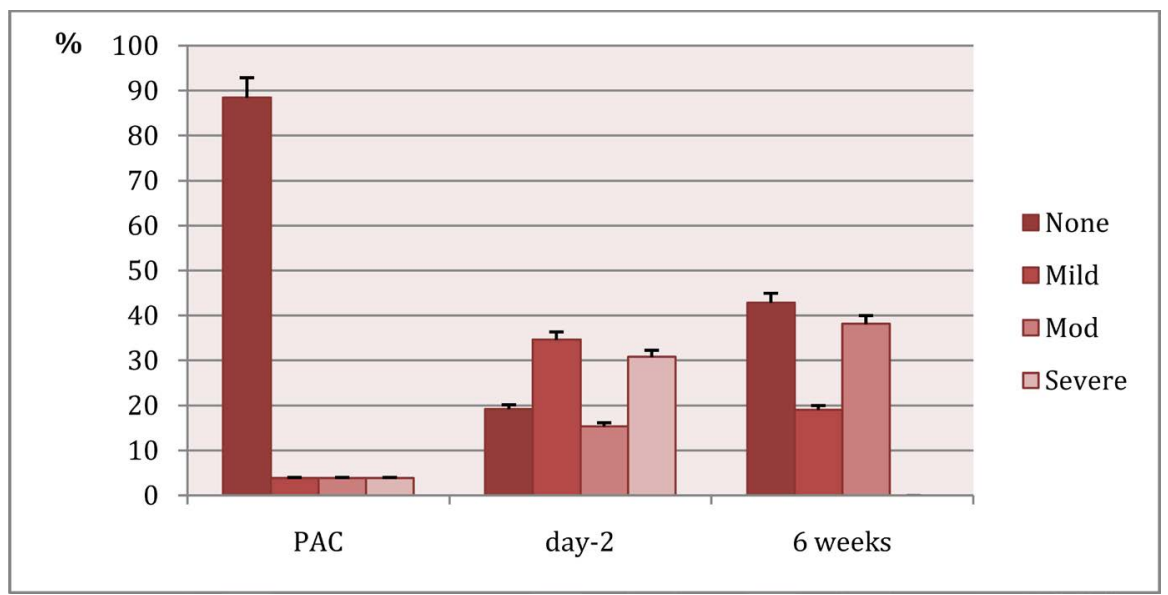

PAC: Pre-admissions clinic.

Figure 2. Rates of different Bazaz dysphagia severities, expressed as percentage of total patients.

modification to soft, minced or puree $(\mathrm{P}=0.06$, linear correlation $\mathrm{P}=0.02)$. There was a linear trend towards increasing number of patients on modified solid diets with increasing day-2 Bazaz severity $(\mathrm{r}=0.95, \mathrm{P}=0.017)$ and Dysphagia short questionnaire score $(r=0.29, \mathrm{P}=0.049)$ only when the solid diet categories were combined. There was no correlation between the different measures and solid modification at 6 weeks or liquid modification at any time interval. Only $8 \%$ of patients were on a modified liquid (day-2).

\subsection{Predictors of Postoperative Dysphagia}

Respiratory comorbidity increased the risk of failing day-2 3-ounce swallow by 32-fold (OR: 32.4; 95\% CI: 1.8 - 587.7; P = 0.019) and scoring moderate or severe on Bazaz by nearly 10-fold (OR: 9.3; 95\% CI: 0.09 - 95.95; P = 0.061). Psychiatric history also increased the risk of failing day-2 3 -ounce swallow by 10 -fold (OR 
10.9; 95\% CI: 1.0 - 123.7; $\mathrm{p}=0.054)$. Demographic factors such as age, gender, BMI and smoking status did not correlate with dysphagia risk, nor did cardiovascular disease or diabetes. Surgical factors such as mode of presentation, high-level or multilevel surgery, operative time and plate apposition were also not associated with dysphagia risk.

\subsection{Interaction between Dysphagia, Hospital LOS and Complications}

Failing day-2 3-ounce swallow test was the only dysphagia measure associated with increased length of stay ( 5 versus 7 days; $\mathrm{P}=0.013$ ), as did suffering a postoperative complication (4.5 versus 7.5 days; $\mathrm{P}=0.006$ ). Cardiovascular comorbidity (40 versus $13 \% ; \mathrm{P}=0.041$ ) and emergency presentation (67 versus $17 \% ; \mathrm{P}=0.017$ ) were associated with increased complication rate, however, the four different dysphagia measures were not.

\section{Discussion}

\subsection{Incidence, Severity and Impact}

Our study findings confirm that the incidence and severity of dysphagia following ACDF depends on the measurement tool used and time interval applied. Knowledge of the incidence, severity and natural history of postoperative dysphagia in an Australasian cohort of patients allows clinicians to better educate, counsel and reassure patients of their expected swallowing outcomes, including in the immediate postoperative period. In our study, dysphagia rate peaked at $85 \%$ on day-2 Dysphagia short questionnaire, with $31 \%$ scoring severe on Bazaz dysphagia scale. Both incidence and severity fell over time, often to near baseline by 6 weeks. We demonstrated that while reports of dysphagia are common, particularly in the immediate postoperative period, this for the most part did not translate into dietary modification. We showed that not only is the novel 3-ounce swallow test a feasible bedside tool, it is also the tool most predictive of dietary modification and increased length of stay following ACDF. The 3-ounce swallow test could therefore potentially stratify at-risk patients, obviate the need for more invasive tests such as videofluoroscopy and fibreoptic endoscopy, and significantly impact discharge planning.

\subsection{Predictors of Dysphagia}

In this prospective study, the most powerful predictor of postoperative dysphagia was respiratory comorbidity, followed by psychiatric history. While psychiatric history has previously been shown to be a risk factor for dysphagia [19], this is the first time that respiratory disease has been specifically assessed. Respiratory and swallowing difficulties often coexist in the general population [20], and it is plausible that surgery may unmask an underlying predisposition to dysphagia in patients with respiratory disease. The presence of respiratory and psychiatric history may therefore identify a group of at-risk patients that may benefit from 
preoperative and early postoperative speech pathology and dietician intervention. A patient's respiratory or psychiatric condition should therefore be optimised prior to ACDF. The presence of inadequately controlled respiratory or psychiatric disease may influence the choice of surgical approach. Furthermore, the lack of an association between plate apposition and postoperative dysphagia, and indirectly the excision of anterior osteophytes, suggests that the pathophysiology of dysphagia extends beyond purely anatomical considerations.

\subsection{Limitations}

The maim limitation to our study was the small sample size. This may partly explain why no significant correlations were demonstrated between traditional risk factors such as high-level and multilevel surgery and postoperative dysphagia. Furthermore, our study was performed in a single institution with relatively short follow-up, although most instances of dysphagia had resolved during follow-up. Despite these shortcomings, we were able to demonstrate statistically significant results in a prospective Australasian cohort of patients, adding to the existing dysphagia literature.

\section{Conclusion}

Dysphagia incidence and severity are highest during the immediate postoperative period, improves in the majority of cases by 6 weeks, and varies according to the measure used, with the 3-ounce swallow test most predictive of dietary modification and length of stay, independent of postoperative complication. Respiratory comorbidity posed the most significant dysphagia risk, followed by psychiatric history. The presence of either of these comorbidities, particularly if associated with failing the 3-ounce swallow test while an inpatient, may identify an at-risk group of patients who require early intervention and close follow-up.

\section{Acknowledgements}

The authors wish to acknowledge the work of the residents, registrars and speech pathologists who assisted in the collection of patient questionnaires and administration of swallow tests.

\section{References}

[1] Joseph, J.R., Smith, B.W., Mummaneni, P.V., La Marca, F. and Park, P. (2016) Postoperative Dysphagia Correlates with Increased Morbidity, Mortality, and Costs in Anterior Cervical Fusion. Journal of Clinical Neuroscience, 31, 172-175. https://doi.org/10.1016/j.jocn.2016.03.011

[2] Rihn, J.A., Kane, J., Albert, T.J., Vaccaro, A.R. and Hilibrand, A.S. (2011) What Is the Incidence and Severity of Dysphagia after Anterior Cervical Surgery? Clinical Orthopaedics and Related Research, 469, 658-665. https://doi.org/10.1007/s11999-010-1731-8

[3] Bazaz, R., Lee, M.J. and Yoo, J.U. (2002) Incidence of Dysphagia after Anterior Cervical Spine Surgery: A Prospective Study. Spine, 27, 2453-2458.

https://doi.org/10.1097/00007632-200211150-00007 
[4] Mchorney, C.A., Robbins, J., Lomax, K., et al. (2002) The SWAL-QOL and SWAL-CARE Outcomes Tool for Oropharyngeal Dysphagia in Adults: III. Documentation of Reliability and Validity. Dysphagia, 17, 97-114. https://doi.org/10.1007/s00455-001-0109-1

[5] Chen, A.Y., Frankowski, R., Bishop-Leone, J., et al. (2001) The Development and Validation of a Dysphagia-Specific Quality-of-Life Questionnaire for Patients with Head and Neck Cancer: The M. D. Anderson Dysphagia Inventory. Archives of Otolaryngology-Head and Neck Surgery, 127, 870-876.

[6] Skeppholm, M., Ingebro, C., Engström, T. and Olerud, C. (2012) The Dysphagia Short Questionnaire: An Instrument for Evaluation of Dysphagia: A Validation Study with 12 Months' Follow-Up after Anterior Cervical Spine Surgery. Spine, 37, 996-1002. https://doi.org/10.1097/BRS.0b013e31823a7a5b

[7] Suiter, D.M. and Leder, S.B. (2008) Clinical Utility of the 3-Ounce Water Swallow Test. Dysphagia, 23, 244-250. https://doi.org/10.1007/s00455-007-9127-y

[8] Cho, S.K., Lu, Y. and Lee, D.H. (2013) Dysphagia Following Anterior Cervical Spinal Surgery: A Systematic Review. Bone \& Joint Journal, 95-B, 868-873. https://doi.org/10.1302/0301-620X.95B7.31029

[9] Nagoshi, N., Tetreault, L., Nakashima, H., et al. (2017) Risk Factors for and Clinical Outcomes of Dysphagia after Anterior Cervical Surgery for Degenerative Cervical Myelopathy: Results from the AOSpine International and North America Studies. The Journal of Bone and Joint Surgery American, 99, 1069-1077. https://doi.org/10.2106/JBJS.16.00325

[10] Zhong, Z.M., Li, M., Han, Z.M., et al. (2016) Does Cervical Disc Arthroplasty Have Lower Incidence of Dysphagia than Anterior Cervical Discectomy and Fusion? $A$ Meta-Analysis. Clinical Neurology and Neurosurgery, 146, 45-51. https://doi.org/10.1016/j.clineuro.2016.04.002

[11] Radcliff, K.E., Bennett, J., Stewart, R.J., et al. (2016) Change in Angular Alignment Is Associated with Early Dysphagia after Anterior Cervical Discectomy and Fusion. Clinical Spine Surgery, 29, 248-254.

[12] Mendoza-lattes, S., Clifford, K., Bartelt, R., Stewart, J., Clark, C.R. and Boezaart, A.P. (2008) Dysphagia Following Anterior Cervical Arthrodesis Is Associated with Continuous, Strong Retraction of the Esophagus. The Journal of Bone and Joint Surgery. American Volume, 90, 256-263. https://doi.org/10.2106/JBJS.G.00258

[13] Kowalczyk, I., Ryu, W.H., Rabin, D., Arango, M. and Duggal, N. (2015) Reduced Endotracheal Tube Cuff Pressure to Assess Dysphagia after Anterior Cervical Spine Surgery. Journal of Spinal Disorders \& Techniques, 28, E552-E558. https://doi.org/10.1097/BSD.0000000000000033

[14] Chin, K.R., Eiszner, J.R. and Adams, S.B. (2007) Role of Plate Thickness as a Cause of Dysphagia after Anterior Cervical Fusion. Spine, 32, 2585-2590. https://doi.org/10.1097/BRS.0b013e318158dec8

[15] Kepler, C.K., Rihn, J.A., Bennett, J.D., et al. (2012) Dysphagia and Soft-Tissue Swelling after Anterior Cervical Surgery: A Radiographic Analysis. The Spine Journal, 12, 639-644. https://doi.org/10.1016/j.spinee.2012.03.024

[16] Jeyamohan, S.B., Kenning, T.J., Petronis, K.A., Feustel, P.J., Drazin, D. and Dirisio, D.J. (2015) Effect of Steroid Use in Anterior Cervical Discectomy and Fusion: A Randomized Controlled Trial. Journal of Neurosurgery: Spine, 23, 137-143. https://doi.org/10.3171/2014.12.SPINE14477

[17] Lee, S.H., Kim, K.T., Suk, K.S., Park, K.J. and Oh, K.I. (2011) Effect of Retropharyngeal Steroid on Prevertebral Soft Tissue Swelling Following Anterior Cervical 
Discectomy and Fusion: A Prospective, Randomized Study. Spine, 36, 2286-2292. https://doi.org/10.1097/BRS.0b013e318237e5d0

[18] Atherton, M., Bellis-Smith, N., Chichero, J.A.Y. and Suter, M. (2007) Texture-Modified Foods and Thickened Fluids as Used for Individuals with Dysphagia: Australian Standardised Labels and Definitions. Nutrition and Dietetics, 64, S53-S76.

[19] Kang, S.S., Lee, J.S., Shin, J.K., Lee, J.M. and Youn, B.H. (2014) The Association between Psychiatric Factors and the Development of Chronic Dysphagia after Anterior Cervical Spine Surgery. European Spine Journal, 23, 1694-1698. https://doi.org/10.1007/s00586-014-3281-3

[20] Steidl, E., Ribeiro, C.S., Gonçalves, B.F., Fernandes, N., Antunes, V. and Mancopes, R. (2015) Relationship between Dysphagia and Exacerbations in Chronic Obstructive Pulmonary Disease: A Literature Review. International Archives of Otorhinolaryngology, 19, 74-79. 